\title{
CHAPEAUX, CASQUETTES ET BÉRETS : QUAND LES INDUSTRIES DISPERSÉES DU SUD COIFFAIENT LE MONDE
}

\author{
Jean-Marc Olivier
}

Dans la société actuelle où l'homme apparaît le plus souvent tête nue, comment imaginer le rôle décisif joué par les couvre-chefs pendant le $\mathrm{XIX}^{\mathrm{e}}$ siècle et la première moitié du $\mathrm{XX}^{\mathrm{e}}$ ? Observer les premières cartes postales suffit pour se persuader de l'ampleur statistique du phénomène, sortir sans chapeau ou sans casquette constitue une faute de goût, voire une provocation, à la Belle Époque. Dans les premières pages du Grand Meaulnes, François raconte l'arrivée solennelle de la nouvelle coiffure de sa mère : " Toute la journée, Millie avait attendu une voiture de la gare qui devait lui apporter un chapeau pour la mauvaise saison. Le matin, elle avait manqué la messe ; et jusqu'au sermon, assis dans le chœur avec les autres enfants, j'avais regardé anxieusement du côté des cloches, pour la voir entrer avec son chapeau neuf ${ }^{1}$ ».

Le chapeau, et tout ce qui s'en rapproche, possède donc de multiples facettes. Son simple rôle de protection contre les intempéries semble vite dépassé par sa fonction de marqueur social ou par sa place de plus en plus essentielle parmi les objets de mode, sans oublier sa dimension politique précoce. Chapeaux, casquettes et bérets alimentent très tôt des dynamiques marchandes subtiles, voire éphémères. Leur volume s'amplifie après 1789 quand ces coiffures se démocratisent. C'est en partant de ces produits aux variations complexes que nous tenterons de décrire les circuits et les réseaux qui stimulent une activité considérable dans la moitié sud de la France. Nous présenterons dans un second temps l'originalité du système de production qui s'impose progressivement, avec une forte dispersion et une « ruralisation » croissante.

\section{Le dynamisme commercial des industries chapelières du Sud}

En fondant notre réflexion sur plusieurs études micro-historiques centrées sur les entreprises chapelières, force est de constater que leur réussite repose largement sur l'intelligence commerciale face à des consommateurs très versatiles ${ }^{2}$.

\section{Faire face à une demande croissante et de plus en plus fluctuante}

La généralisation du port du chapeau appartient au $\mathrm{XIX}^{\mathfrak{e}}$ siècle. Cette période débute par un certain renouvellement du bon goût dans les élites. Au tricorne du XVIII ${ }^{\mathrm{e}}$ siècle

\footnotetext{
${ }^{1}$ Alain-Fournier, Le Grand Meaulnes, Paris, Fayard, 1971 (première édition en 1913), 266 p., p. 15.

${ }^{2}$ Lors de ces dix dernières années, cinq mémoires de maîtrise soutenus à l'université de Toulouse 2-Le Mirail ont été consacrés à la chapellerie du Sud-Ouest. À cela s'ajoutent les recherches réalisées dans le cadre de l'atelier « Industries et industrialisations » du laboratoire FRAMESPA (UMR 5136). Ces travaux ont mobilisé des sources fines comme les inventaires après décès, les dossiers de faillites et les archives privées des entreprises afin de dépasser le simple constat quantitatif partiel et partial que l'on établit à partir des sources sérielles du type recensements ou enquêtes industrielles.
} 
succède le haut-de-forme ${ }^{3}$. Chez les femmes, dans les années 1820 , charlottes, toques et turbans cèdent soudain la place à d'énormes chapeaux couverts de rubans et de plumes ${ }^{4}$. Cependant, les variations de style ne vont plus cesser désormais et elles s'accompagnent de modifications de détails qui contribuent à un renouvellement permanent. Ainsi, des variantes du haut-de-forme sont obtenues en jouant sur la taille des calottes et des bords. Les larges bords avec très petites calottes à sommet plat prédominent, puis les calottes basses s'affirment dans les années 1840 jusqu'à l'apparition du haut-de-forme à calotte rabattable en 1848 .

À la diversité des formes et des décors s'ajoute celle des matières, on en distingue trois principales : le feutre, la paille et le tissu. À celles-ci correspondent trois fabrications assez distinctes. Un changement de mode portant sur les matériaux de base peut donc avoir des conséquences dramatiques pour l'industriel qui ne réalise qu'un seul type de produit. Ainsi, dans les années 1830, les Toulousains abandonnent le chapeau de feutre car ils lui préfèrent celui en tissu de soie $^{5}$; après un retour en grâce, le feutre connaît une nouvelle éclipse à la fin des années 1870. Des changements saisonniers rythment également le choix des matières, le chapeau de paille, de plus en plus léger, triomphe en été à partir des années 1820. Sa forme peut varier à l'infini et l'entreprise Herment de Septfonds dans le Tarn-etGaronne propose 900 modèles différents au début des années $1880^{6}$. À la Belle Époque règnent le canotier et le panama, l'aviateur Santos-Dumont lance alors la mode du panama mou dont il ne se sépare jamais.

Au-delà des modes, ces coiffures sont aussi des marqueurs sociaux. À l'image du pharmacien de Knoch ${ }^{7}$ qui se désole de ne pas pouvoir offrir à sa femme des chapeaux dignes de son rang, chacun évalue la position de l'autre en fonction de son couvre-chef. Toutefois, les classements simples du premier $\mathrm{XIX}^{\mathrm{e}}$ siècle - béret du paysan du Sud-Ouest, casquette de l'ouvrier et chapeau du bourgeois - se révèlent de moins en moins opératoires. Plus particulièrement, le succès du béret traverse toutes les strates de la société.

Attribut des ruraux du Béarn et du Pays Basque depuis le $X V^{\mathrm{e}}$ siècle, le «berret », mot béarnais, ne se diffuse dans la langue française qu'à partir du XIX $\mathrm{X}^{\mathrm{e}}$ siècle $^{8}$. Ainsi, dès 1829, un marchand de Nay (Béarn) remarque « un béret porté par une princesse du

${ }^{3} \mathrm{Mc}$ Dowell (Colin), Le chapeau et la mode des origines à nos jours. Fascination, charme, rang et style, Paris, CELIV, 1994, $224 \mathrm{p}$.

${ }^{4}$ Ibidem

${ }^{5}$ Archives municipales de Toulouse, Exposition des produits des Beaux Arts et de l'Industrie dans les galeries du Capitole à Toulouse le 20 juin 1835, Toulouse, Henault, 1835, 220 p., p. 100.

${ }^{6}$ Ortiz (Vincent), Un chapeau de paille de Septfonds: la chapellerie dans le canton de Caussade au XIX siècle, mémoire de maîtrise, université de Toulouse 2-Le Mirail, 2004, 319 p., pp. 84-85

Romain (Jules), Knock, 1923 (première représentation de la pièce à Paris), acte II, scène 3. Le pharmacien Mousquet (se plaignant de la faiblesse de ses revenus imputable au faible nombre d'ordonnances prescrites par le prédécesseur de Knoch): « Oui, certes. Toute question d'argent à part, il y a conscience à se laisser glisser ainsi au-dessous du ferblantier et de l'épicier. Je vous assure, docteur, que ma femme serait bien empêchée de se payer les chapeaux et les bas de soie que la femme du ferblantier arbore semaine et dimanche ».

${ }^{8}$ Maysonnave (Jean), Le béret. Essai pour une histoire, Pau, Mercure-Segré, 1968, 50 p., p. 13 et p. 7. 
sang dans un pèlerinage à Betharram ${ }^{9}$. Cependant, l'ascension du béret se trouve surtout favorisée par les militaires. Dès le $\mathrm{XVIII}^{\mathrm{e}}$ siècle, les régiments basques, biscayens et Pyrénéens, appelés cantabres, portent un béret. Murat en coiffe sa garde personnelle, puis le général Bosquet en réclame pour ses troupes d'Algérie dès $1831^{10}$. Les commandes affluent à partir des années 1830 car il devient l'attribut des chasseurs à pied puis celui des chasseurs alpins après 1871. Le service militaire obligatoire en vulgarise davantage l'emploi civil, désormais beaucoup d'ouvriers et de paysans le portent en dehors du Sud-Ouest. Il connaît aussi les honneurs de la mode par l'intermédiaire du sport. Dans les années 1920, les tennismen Jean Borotra et Suzanne Lenglen l'arborent dans leurs succès internationaux, tandis que les joueurs de rugby du Stade toulousain, François Borde et Adolphe Jauréguy, l'exploitent jusque sur le terrain comme une feinte en le faisant passer pour le ballon ${ }^{11}$.

Finalement, la mode féminine s'empare du béret en l'utilisant sans cuir avec des coloris très variés. Souvent, les modistes le transforment en «bibis » bon marché avec des pinces, des piqûres, des fronces ou des bordures. Les années 1930 marquent l'apogée du béret que beaucoup de groupes politiques cherchent à s'approprier, à l'image des Croix-de-Feu du colonel de La Rocque. En définitive, jouant sur les matières et les décors, les chapeliers du Sud réussissent à répondre aux goûts changeants de la clientèle et pour cela ils s'appuient sur leurs réseaux de fournisseurs constamment étoffés.

\section{Gérer des approvisionnements de plus en plus complexes}

Les matières premières représentent en moyenne $50 \%$ du prix de revient d'un chapeau $^{12}$, leur maîtrise apparaît donc capitale. Déjà, à la fin du XVI ${ }^{\mathrm{e}}$ siècle, la mode du chapeau de castor avait déclenché une forte spéculation sur les fourrures canadiennes. Toutefois, dans un premier temps, les petits producteurs méridionaux du début du $\mathrm{XIX}^{\mathrm{e}}$ siècle cherchent à valoriser les ressources locales comme le poil de lapin pour les feutres ou les plumes d'oies et de canard pour les décors. La paille de seigle du Quercy et du Tarn-etGaronne, rigide et blanche, alimente les chapeliers de Septfonds qui l'achètent tressée ; ils se heurtent à leurs rivaux lyonnais qui viennent également s'approvisionner sur les marchés d'hiver de Puylaroque, Montpezat et Lalbenque ${ }^{13}$, voire sur celui de Septfonds. Dans ce dernier village, tresseurs et chapeliers locaux s'entendent entre eux avant l'ouverture du marché, ce qui provoque une protestation des « étrangers » en $1869^{14}$.

La nécessité d'afficher une offre plus variée - la paille de seigle indigène ne servant qu'à l'article commun - engendre des importations de plus en plus élaborées. Le paillasson suisse, le picot de Toscane et la paille jaspée de Belgique ne suffisent pas ${ }^{15}$. En 1869, Fortuné Cantecor, chapelier du village de Septfonds, remarque chez un client londonien

\footnotetext{
${ }^{9}$ Ibidem, p. 19.

${ }^{10}$ Ibidem, p. 23

${ }^{11}$ Ibidem, p. 24

${ }^{12}$ Jullian (Antoine), Itinéraires et changements sociaux dans la chapellerie espérazanaise du $X I X^{e}$ siècle, mémoire de maîtrise, université de Toulouse 2-Le Mirail, 2004, 90 p., p. 17.

${ }^{13}$ Thomas (Jack), Le temps des foires. Foires et marchés dans le Midi toulousain de la fin de l'Ancien Régime à 1914, Toulouse, Presses universitaires du Mirail, 1993, 407 p., p. 166.

${ }^{14}$ Ortiz (Vincent), Un chapeau de paille de Septfonds..., ouv. cité, p. 104.

${ }^{15}$ Ibidem, pp. 113-114.
} 
des tresses de Chine, jusque-là monopole des Anglais ; aussitôt, il en organise l'importation à grande échelle et ces tresses d'Extrême-Orient représentent rapidement $80 \%$ de celles utilisées par la chapellerie de paille tarn-et-garonnaise ${ }^{16}$. En 1889, le même homme envoie un membre de sa famille à Batavia ouvrir un comptoir destiné à importer des cloches de chapeaux javanaises, en rotin, plus légères et plus minces que toutes celles qui existent. L'année suivante, Fortuné Cantecor décède, laissant plus de 600000 francs à ses héritiers ${ }^{17}$. Cette fortune en fait l'égal de beaucoup d'industriels français, même s'il ne possède pas de grande usine, son génie étant d'abord commercial.

À une échelle plus modeste, les chapeliers et modistes toulousains s'insèrent également dans des réseaux complexes. Deux sources particulières autorisent une approche plus fine de ce phénomène difficile à repérer: les bilans de faillites et les déclarations d'incidents relatifs au transport des marchandises sur le canal du Midi. Cette dernière source s'avère très riche. En effet, les archives du canal du Midi ${ }^{18}$ relatives à la navigation accélérée conservent un «État des retenues exercées pour manques, avaries et retards, dressé pour chaque trimestre dans les sources comptables $\gg{ }^{19}$. Ces actes sous seing privé présentent successivement : la compagnie chargée du transport, la ville de départ, même si elle n'est pas située sur une voie d'eau, la ville d'arrivée, la date et surtout la nature des marchandises transportées avec les dégâts qu'elles ont subis. Ces documents, qui constituent un échantillonnage de plus de 3000 incidents déclarés entre 1826 et 1858, démontrent une circulation intense des objets et des matières utilisées par les petites industries toulousaines. Ainsi, la chapellerie de la ville rose importe des peaux de castors de Grenoble, dès 1844, des rubans de Saint -Étienne en 1855, des poils de lapins de Marseille la même année et des « chinchillas frisés » d'Elbeuf en $1856^{20}$. Les bilans de faillites démontrent l'existence de fournisseurs européens, en particulier l'entreprise Goudal de Toulouse qui importe des éléments de chapeaux de Londres et d'Eupen en Allemagne, puis des cuirs à chapeaux de Bruxelles à partir de $1888^{21}$.

Les chapeliers du Sud de la France deviennent donc de fervents défenseurs du libre-échange afin de disposer de toutes ces matières aux meilleurs prix. De cette façon, ils obtiennent en 1892 une réduction du tarif sur les «tresses de paille, d'écorce et de bois blanc », leur taxation passant de cinq francs à trois francs les cent kilogrammes ${ }^{22}$. Cette prise de position s'explique aussi par la peur de mesures de rétorsions sur les exportations de chapeaux terminés si la France se montre trop protectionniste.

\footnotetext{
${ }^{16}$ Ibidem, pp. 111-114.

${ }^{17}$ Archives départementales du Tarn-et-Garonne, 3Q 1457, folios 24 à 28, mutation par décès d'André Désiré Fortuné Cantecor, 6 novembre 1890.

${ }^{18}$ Archives du canal du Midi, gérées par les Voies Navigables de France, Direction générales du Sud-Ouest, 26 Port Saint-Etienne, Toulouse, documents relatifs à la comptabilité (1826$1858)$.

19 Jeanjean (Patricia), La navigation accélérée sur le canal du Midi (1826-1858), mémoire de maîtrise, université de Toulouse 2-Le Mirail, 2002, deux tomes, 206 p. et 177 p.

${ }^{20}$ Ibidem, p. 46, p. 149 , p. 151 , p. 147 et p. 170.

${ }^{21}$ Passama (Julie), La chapellerie toulousaine au XIX $X^{e}$ siècle, mémoire de maîtrise, université de Toulouse 2-Le Mirail, 2002, 266 p., p. 115

${ }^{22}$ Ortiz (Vincent), Un chapeau de paille de Septfonds..., ouv. cité, pp. 113-114
} 


\section{L'élargissement constant des réseaux commerciaux}

Dès le Second Empire, stimulée par la réussite de Joseph Maraval, la chapellerie albigeoise spécialisée dans les feutres exporte vers l'Espagne, la Roumanie, la Russie, l'Égypte et l'Allemagne ${ }^{23}$. À la même époque, les établissements de la haute vallée de l'Aude, plus particulièrement ceux d'Espéraza, connaissent un bel essor et diffusent leurs chapeaux de feutre de laine jusqu'en Amérique ${ }^{24}$. La chapellerie toulousaine participe à ces flux; dès les années 1850, Hispa exporte ses chapeaux de feutre dans toute l'Europe occidentale et en Amérique latine, plus particulièrement au Brésil, au Chili et au Venezuela ${ }^{25}$. Il écoule également ses marchandises vers la Louisiane en utilisant le port de la Nouvelle-Orléans ${ }^{26}$. Les quantités sur lesquelles portent ces transactions demeurent difficiles à saisir, cependant, une lettre de 1863 du président de la chambre de commerce au préfet fournit un ordre de grandeur en relevant que « la crainte de la guerre a conduit à refuser une fourniture de 150000 francs pour Buenos Aires ${ }^{27}$.

De leur côté, les bérets réalisés en série à Nay et à Oloron-Sainte-Marie suivent les circuits traditionnels du commerce méridional. Ils remportent d'ailleurs un vif succès en Espagne où ils apparaissent dans chaque camp lors des guerres carlistes dès la première moitié du XIX ${ }^{\mathrm{e}}$ siècle $^{28}$. Plus tard, pendant la Guerre Civile, bérets rouges et bérets noirs s'affrontent. L'Afrique du Nord constitue également un débouché facile, car il suffit de modifier quelques réglages de machine pour passer du béret à la chéchia ${ }^{29}$. Enfin, la coiffure basque et béarnaise franchit l'Atlantique et, en 1936, aux jeux olympiques de Berlin, la délégation américaine défile coiffée de bérets, tandis que la française arbore des chapeaux de paille $^{30}$. Cette dernière coiffure, produite massivement dans le Tarn-et-Garonne, se heurte à une forte concurrence internationale. En effet, la Toscane, le canton d'Argovie en Suisse, le comté de Bedford en Angleterre, la Saxe, la région de Liège et la Chine, produisent également des chapeaux de paille ${ }^{31}$. Pourtant, les chapeliers Septfontois et Caussadais réussissent à pénétrer les marchés anglais et italien, puis le reste de l'Europe, et, plus traditionnellement, l'empire colonial.

Cette réussite commerciale repose sur une forte présence directe à tous les niveaux afin d'éliminer les intermédiaires. Ainsi, les chapeliers de Septfonds, qui dépendaient

${ }^{23}$ Corduries (Sylvie), L'industrie à Albi au XIX siècle, 1800-1896, mémoire de maîtrise, université de Toulouse 2-Le Mirail, 1996, 153 p., p. 105

${ }^{24}$ Christol (Corinne), L'industrie chapelière dans la haute vallée de l'Aude aux XIX et XX siècles, mémoire de maîtrise, université de Toulouse 2-Le Mirail, 1995, 117 p., p. 13.

${ }^{25}$ Archives municipales de Toulouse, Exposition des Beaux Arts et de l'Industrie à Toulouse en 1858, Toulouse, Douladoure frères, 1859, 535 p., p. 183.

${ }^{26}$ Ibidem.

${ }^{27}$ Archives départementales de la Haute-Garonne, 12 M 34, année 1863.

${ }^{28}$ Maysonnave (Jean), Le béret..., ouv. cité, p. 24.

${ }^{29}$ Ibidem. De plus, il existe une tradition française d'exportation des chéchias vers les Échelles du Levant (cf. Maitte (Corine), «Adapter les produits, jouer sur les marchés. La fabrication des chéchias, XVIII ${ }^{\mathrm{e}}$-XIX ${ }^{\mathrm{e}}$ siècles », dans Fontana (Giovanni Luigi) et Gayot (Gérard) (éd.), La laine : produits et marchés (XIII ${ }^{e}-X X^{e}$ siècles), Padoue, 2004, 1228 p., pp. 1115-1142)

${ }^{30}$ Ibidem, p. 25

${ }^{31}$ Gebelin (Jacques), Une grande industrie du Bas-Quercy. Les chapeaux de paille de Septfonds et Caussade, Bordeaux, Feret et fils, 1895, 16 p., p. 14. 
du marché anglais de Luton pour les tresses d'Extrême-Orient, s'en émancipent progressivement; en 1894, deux tiers des tresses sont importés directement ${ }^{32}$. À l'exportation, les commissionnaires extérieurs, parisiens ou lyonnais, exercent encore leur influence pour les expéditions lointaines, mais les producteurs méridionaux contrôlent personnellement les marchés européen et africain. Pour arriver à cette indépendance, ils ont recours à de nombreux voyageurs de commerce particulièrement fidèles car souvent recrutés dans la famille proche ou dans le village. Cette émancipation semble précoce dans le domaine du feutre, elle se révèle plus tardive dans la chapellerie de paille. C'est pourquoi, d'après les listes nominatives de recensement, Septfonds ne connaîtrait qu'un seul voyageur de commerce en $1881^{33}$, puis ce chiffre s'élèverait à 38 en $1901^{34}$. Toutefois, la persistance d'une forte pluri-activité agrochapelière peut masquer l'existence de vendeurs-paysans qui ne prennent la route que pendant l'hiver ${ }^{35}$. Ainsi, d'autres sources parlent de plus de deux cents voyageurs de commerce dans les communes de Caussade et Septfonds ${ }^{36}$.

Beaucoup d'entreprises établissent leur réussite sur leur habileté commerciale, leur présence auprès des détaillants et leur capacité à achalander leurs catalogues. Exemple extrême, la société «Rey Cousins et $C^{\mathrm{ie}}$ 》 comporte quinze voyageurs et employés de commerce sur un total de vingt-sept personnes ${ }^{37}$. Ces représentants emmènent dans leurs bagages les échantillons des nouvelles collections, réalisés dans le plus grand secret, par les meilleures ouvrières au début de l'été, pour sillonner la France et l'Europe ${ }^{38}$. Fortuné Cantecor, à son décès en 1890 , dispose d'un réseau très dense de clients métropolitains. Celuici couvre au moins 49 départements, y compris les plus éloignés comme la Manche, la BasseSeine ou la Meurthe-et-Moselle ${ }^{39}$. Les archives privées attestent également l'existence de propositions spontanées émanant d'expatriés intéressés par le négoce. Voici un extrait de celle reçue par le chapelier Herment de Septfonds : « (...) J'ai l'honneur de vous confirmer une lettre postée en janvier dernier et restée sans réponse. Je me permets de vous prier à nouveau de vouloir bien me faire connaître une décision en ce qui concerne mes offres de vous représenter en Roumanie où vos articles sont considérablement rentables, M. Goldstein, Braila le 27 septembre $1910 »{ }^{40}$. Les plus petits établissements n'hésitent pas à adopter une démarche commerciale active et beaucoup de modistes toulousaines effectuent des voyages à Paris, accompagnées de leur "première », c'est-à-dire la meilleure ouvrière ou la plus

\footnotetext{
${ }^{32}$ Ibidem, p. 15

${ }^{33}$ Archives départementales du Tarn-et-Garonne, 6M 113

${ }^{34}$ Ibidem, 6M 178

${ }^{35}$ Les listes nominatives des dénombrements du XIX ${ }^{\mathrm{e}}$ siècle n'enregistrant qu'une profession, les pluri-actifs (paysans-tisserands, paysans-horlogers, paysans-lunetiers, paysans-chapeliers etc.) préfèrent souvent se déclarer agriculteurs, ou propriétaires, dans cette France encore très rurale où la terre reste médiatrice des rapports sociaux. Ce comportement se traduit par une sous-estimation des activités industrielles rurales dispersées.

${ }^{36}$ Doumerc, Industrie, commerce et voies de communication du département du Tarn-etGaronne, Montauban, Edouard-Forestié imprimeur, 1902, 53 p., p. 19

${ }^{37}$ Ortiz (Vincent), Un chapeau de paille de Septfonds..., ouv. cité, p. 90.

${ }^{38}$ Ibidem, p. 91

${ }^{39}$ Ibidem, p. 101

${ }^{40}$ Ibidem, p. 92, archives privées Mme Fontayne.
} 
ancienne ${ }^{41}$. Ces expéditions permettent de visiter les clients et de se tenir informé des premiers frémissements de la mode.

Cette présence humaine très personnalisée se double d'une participation aux grandes expositions et d'une volonté de s'intégrer dans les réseaux de transport les plus modernes. Dès 1867, l'entreprise Rey de Septfonds obtient une médaille de bronze à l'exposition universelle de Paris, puis une d'argent à celle d'Amsterdam en 1883 et, enfin, une médaille d'or à Paris en $1900^{42}$. La démarche de participation peut être collective, ainsi, Jean Labat, ouvrier chapelier à Caussade, reçoit une aide de la municipalité s'élevant à 150 francs pour se rendre à l'exposition universelle de Paris de 1889; en 1900, ce sont trois de ses collègues de Septfonds qui bénéficient du même type de soutien pour rejoindre la capitale ${ }^{43}$. L'obtention d'une gare pour Caussade sur la ligne Montauban-Cahors ouverte en 1884 confirme cette dynamique collective. Cette station se montre rapidement très rentable, expédiant chaque année 75000 colis d'une valeur de 3500000 francs dès le début du XX siècle $^{44}$. En 1913, une gare spécifique est ouverte dans le village chapelier originel de Septfonds, les industriels obtiennent son agrandissement dans les années 1920 avec la réalisation d'un abri destiné à protéger leurs marchandises. À Espéraza, dans la haute vallée de l'Aude, le roulage ne constitue qu'une solution temporaire. Une jonction par voie ferrée est finalement établie avec Carcassonne en 1878, malgré les difficultés liées au relief ${ }^{45}$. En 1913, cette gare assure l'importation, devenue nécessaire, de mille tonnes de laine par an, et l'expédition de 35000 colis $^{46}$.

Au total, la chapellerie méridionale participe brillamment à l'équilibrage de la balance commerciale française du $\mathrm{XIX}^{\mathrm{e}}$ siècle. Ceci s'avère particulièrement vrai pour les chapeaux de paille où le Sud joue un rôle majeur; dès 1890, les exportations de ce secteur s'élèvent à plus de 6700000 francs alors que les importations ne représentent que 3100000 francs $^{47}$. L'excédent se confirme avec les chapeaux de feutre, spécialité de Toulouse et d'Espéraza, où il atteint plus de quatre millions de francs ${ }^{48}$. Les performances de cette chapellerie du Sud soumise à une forte concurrence nationale et étrangère suscitent l'étonnement, car elles ne reposent pas sur la concentration de l'activité dans de vastes usines urbaines.

\section{Souplesse des systèmes de production chapeliers dispersés}

\footnotetext{
${ }^{41}$ Passama (Julie), La chapellerie toulousaine au XIX $X^{e}$ siècle, mémoire de maîtrise, université de Toulouse 2-Le Mirail, 2002, 265 p., p. 188.

${ }^{42}$ Ortiz (Vincent), Un chapeau de paille de Septfonds..., ouv. cité, pp. 94-95.

${ }^{43}$ Archives départementales du Tarn-et-Garonne, 8M 1331-1332.

${ }^{44}$ Daux (Camille), Septfonds en Quercy (Tarn-et-Garonne), Montauban, Forestié, 1900, 77 p., p. 67.

${ }^{45}$ Jullian (Antoine), Itinéraires et changements sociaux dans la chapellerie espérazanaise du $X I X^{e}$ siècle ..., p. 43

${ }^{46}$ Ibidem, p. 65.

${ }^{47}$ Revue de la chapellerie, $\mathrm{n}^{\circ} 3,1890$, p. 137.

${ }^{48}$ Ibidem.
} 
La nécessité de proposer une gamme très variée de produits finis, régulièrement renouvelés, offre un terrain plus favorable à la petite entreprise. Cette dernière se montre plus performante que la mécanisation complète conduite dans des usines citadines. Néanmoins, l'archaïsme apparent des structures éclatées de production demeure trompeur. En réalité, des évolutions importantes aboutissent à des gains de productivité très élevés qui condamnent le petit artisanat traditionnel. La chapellerie méridionale change de visage au tournant du Second Empire et répond à l'explosion de la demande en mobilisant sa main-d'œuvre rurale et en s'appuyant sur les potentialités techniques de ses capitaines d'industrie.

\section{Petite taille des établissements et « ruralisation »}

À l'échelle nationale, une décentralisation de l'industrie du chapeau, de Paris vers la province, se produit dès le milieu du $\mathrm{XIX}^{\mathrm{e}}$ siècle. De nombreuses villes se lancent alors dans cette activité en occupant des créneaux moins nobles. Ce mouvement s'accélère encore entre 1890 et 1914. Beaucoup d'artisans locaux franchissent le pas et deviennent de petits fabricants à Lyon, Marseille, Aix, Bordeaux, Nancy, Épinal, puis Rennes, Roubaix, Lille, Strasbourg, Grenoble, Avignon, Toulon et Toulouse... La plupart de ces établissements restent modestes et fragiles, ils réalisent quelques bons coups commerciaux, mais ne disposant pas de capitaux importants, ils sont emportés par la première difficulté.

Maintes villes du Sud connaissent alors une décadence irrémédiable de leur chapellerie à la fin du XIX ${ }^{\mathrm{e}}$ siècle, comme l'observe Paul Masson, en 1926, dans son encyclopédie départementale sur les Bouches-du-Rhône : " À l'exposition de 1900 on avait constaté le déclin de la fabrication des chapeaux de feutre à Aix comme dans plusieurs villes de France où elle florissait auparavant, à Albi notamment. À Bordeaux, à Dijon, elle avait même disparu. Mais si le nombre des chapeaux fabriqués avait fortement diminué, on affirmait que la qualité avait remplacé la quantité (...). Avec Bourg-de-Péage, Albi, Anduze, Aix excellait encore dans la fabrication des chapeaux de poil souple (...). On félicitait aussi les Aixois d'avoir exhibé une variété infinie de chapeaux portés sous toutes les latitudes, tandis qu'on reprochait à ceux d'Albi et d'Anduze d'avoir apporté une extrême sobriété dans le nombre de leurs modèles. C'était la dernière exposition où la chapellerie aixoise devait figurer avec honneur: les fabricants ne surent pas imposer l'emploi des machines aux ouvriers habitués au travail à la main. Des deux maisons marquantes en 1900, une seule, la maison Milliat, subsistait en 1914 (...). Pour Marseille, la disparition de l'industrie des chapeaux de feutre, une des gloires de la ville au XVIII ${ }^{\mathrm{e}}$ siècle, était un fait accompli avant 1900, mais on avait vu paraître la fabrication des casques coloniaux ou coiffures insolaires pratiquées par plusieurs maisons. Deux autres industries anciennes étaient restées vivantes, celle des casquettes en tous genres, spécialement des casquettes d'uniformes pour la marine ou les diverses administrations (..), et celle des chapeaux de paille (...). Ces fabriques écoulaient une partie de leur production au-dehors, notamment sur le littoral méditerranéen ${ }^{49}$.

49 Masson (Paul) (dir.), Les Bouches-du-Rhône, encyclopédie départementale, deuxième partie : Le bilan du XIX siècle, tome VIII : Le mouvement économique : l'industrie, chapitre $\mathrm{X}$ : La fin du XIX siècle et les débuts du XX (1890-1914). Les industries secondaires, pp. 291-292 (chapitre rédigé par Paul Masson, professeur à l'université d'Aix-Marseille), Marseille, archives départementales des Bouches-du-Rhône, 1926. 
À Toulouse, le déclin semble différé, pourtant la concentration de la main-d'œuvre s'opère très lentement et tardivement, avec des hésitations. Le nombre moyen d'ouvriers pour chaque fabricant progresse peu, de six en 1821 à quatorze en 1911, de plus cela ne signifie pas qu'ils soient obligatoirement rassemblés sur un site unique ${ }^{50}$. Seule une production simplifiée, comme la casquetterie, aboutit à des regroupements significatifs avec une moyenne de 42 ouvriers par patron. En 1911, les 1200 chapeliers, modistes et casquetiers recensés dans Toulouse demeurent extrêmement dispersés ${ }^{51}$. Les ateliers rassemblant plus de dix ouvriers sont exceptionnels. L'essentiel des travailleurs s'agglutine dans la vieille ville. De ce monde de la fabrique dispersée émerge constamment de nouveaux petits entrepreneurs, mais leur existence est souvent éphémère. Entre 1890 et 1914, quinze chapeliers et vingt-trois modistes font faillite, victimes du crédit à la clientèle, de la concurrence ou des exigences des grands magasins, et surtout de l'arrivée de nouveaux producteurs redoutables : les ruraux ${ }^{52}$.

En effet, la féminisation du travail urbain a abaissé les coûts de production, mais pas dans une proportion suffisante ${ }^{53}$. L'essor des centres ruraux se fonde alors sur l'utilisation d'une main-d'œuvre abondante et très bon marché. Ces paysans-chapeliers se contentent de salaires très bas car ils les assimilent à un complément de revenu obtenu pendant les temps morts de l'activité agricole. Ces pluri-actifs présentent également l'avantage d'accepter le caractère saisonnier de la chapellerie, l'arrêt d'une fabrication n'implique pas leur mise au chômage ou leur licenciement. Certains patrons toulousains le comprennent et délocalisent une partie de leur production vers les campagnes environnantes. C'est le cas de Raymond Laffont, inventeur du «casque à ventilation aérifaire », qui s'installe dans le village de Septfonds (Tarn-et-Garonne) pour développer sa production à partir de $1900^{54}$. Toutefois, à cette date, les pôles ruraux ont conquis leur indépendance technique et commerciale, ils ne se contentent plus d'une simple sous-traitance pour des négociants citadins.

Il existe ainsi plusieurs nébuleuses rurales dynamiques qui l'emportent progressivement sur les centres chapeliers urbains au fil du $\mathrm{XIX}^{\mathrm{e}}$ siècle. À Septfonds, les simples tresseurs de paille se muent en capitaines d'industrie et dominent le secteur du chapeau de paille. L'activité essaime vers les localités voisines, en particulier le chef-lieu de canton, Caussade, bourg de 4000 habitants en 1856. Mais Septfonds demeure le centre donneur d'ordres, ce village progresse de 800 habitants au début du $\mathrm{XIX}^{\mathrm{e}}$ siècle à près de 1400 en $1872^{55}$. Le maire déclare dès 1871 que toutes les femmes et filles sont occupées par la chapellerie, dans des ateliers, à domicile, ou dans la rue devant leurs maisons ${ }^{56}$. La haute vallée de l'Aude connaît le même succès avec le chapeau de feutre de laine, surtout à partir de

${ }^{50}$ Passama (Julie), La chapellerie toulousaine au XIX $X^{e}$ siècle, mémoire de maîtrise, université de Toulouse 2-Le Mirail, 2002, 265 p., p. 35 et p. 158.

${ }^{51}$ Ibidem, pp. 156-160.

${ }^{52}$ Ibidem, p. 176

53 À Toulouse, la proportion de femmes dans les différents métiers de la chapellerie (chapelières, modistes, casquetières) progresse de $21 \%$ en 1821 à $92 \%$ en 1911 (Ibidem, p. 58 et p. 181).

${ }^{54}$ Passama (Julie), La chapellerie toulousaine ..., ouv. cité, p. 192.

${ }^{55}$ Ortiz (Vincent), Un chapeau de paille de Septfonds..., ouv. cité, p. 64, « 674 individus, sur une population de 1369, sont impliqués à différents degrés dans l'activité chapelière ».

${ }^{56}$ Archives départementales du Tarn-et-Garonne, 10M 1410. 
$1855^{57}$. Pendant longtemps la dispersion prédomine avec des ateliers occupant généralement entre dix et vingt personnes ${ }^{58}$. En 1913, le bourg d'Espéraza, devenu la capitale française de ce type de coiffure, compte désormais plus de 2000 habitants et emploie 1500 ouvriers chapeliers répartis entre quatorze usines ${ }^{59}$. Néanmoins, une partie de ces ouvriers continue de travailler à domicile. De plus, le recrutement touche progressivement les villages voisins de $\mathrm{Fa}$ ou Rouvenac, puis les bourgs de Couiza, Quillan, Chalabre et Limoux où se développent des activités de production ${ }^{60}$.

Pour atteindre cette indépendance puis dominer le marché, les entrepreneurs ruraux ont su acquérir des compétences techniques. Ainsi, les Septfontois attirent des contremaîtres grenoblois et lyonnais puis des ouvriers italiens, dès les années $1840^{61}$. Cette démarche aboutit à la réalisation des premiers chapeaux de paille apprêtés, tissés et enrichis de garnitures, donc directement commercialisables, en $1849^{62}$. Cependant, le succès de ces nébuleuses rurales réside aussi dans leur organisation très élaborée du travail dispersé.

\section{Division des tâches, paiement aux pièces et travail à domicile}

À la fin du XVIII ${ }^{\mathrm{e}}$ siècle, la chapellerie demeurait majoritairement une activité de type artisanal, la plupart des observateurs estimant qu'un bon ouvrier ne pouvait réaliser que deux chapeaux par jour ${ }^{63}$. L'essor de la demande, au fur et à mesure du XIX $X^{\mathrm{e}}$ siècle, stimule les initiatives. Celles-ci se révèlent innombrables car l'organisation d'une production chapelière à grande échelle ne nécessite pas beaucoup de capitaux, surtout dans le monde rural. L'aménagement d'une pièce dans la maison d'habitation suffit pour créer un atelier central car l'essentiel repose sur l'élaboration d'une division intelligente du travail avec une circulation efficace des matières premières et des chapeaux en cours d'élaboration. Dans la chapellerie de paille septfontoise, la confection de la tresse constitue la première étape, puis les couseuses réalisent le «bouton », point de départ de la "cloche », nom donné au chapeau brut sans forme, également cousu en cercles concentriques. Ces opérations, totalement manuelles à l'origine, mobilisent les bergères du canton de Caussade qui les réalisent en gardant leur troupeau, voire en marchant. Les hommes interviennent ensuite pour apprêter l'ébauche avec de la colle, la sécher par étuvage puis la blanchir avec du souffre et la dresser grâce à des petites presses agissant sur des moules en métal. Enfin, les femmes, «las garnièras », reprennent le chapeau nu pour y ajouter les garnitures constituées d'étoffes, de rubans ou de cuir ${ }^{64}$.

${ }^{57}$ Jullian (Antoine), Itinéraires et changements sociaux dans la chapellerie espérazanaise du $X I X^{e}$ siècle ..., ouv. cité, p. 9.

${ }^{58}$ Ibidem, pp. 43-44.

${ }^{59}$ Ibidem, p. 65.

${ }^{60}$ Christol (Corinne), L'industrie chapelière dans la haute vallée de l'Aude aux XIX et XX siècles, mémoire de maîtrise, université de Toulouse 2-Le Mirail, 1995, 117 p., p. 13.

${ }^{61}$ Ortiz (Vincent), Un chapeau de paille de Septfonds..., ouv. cité, p. 86.

${ }^{62}$ Ibidem.

${ }^{63}$ Vial (Jean), La coutume chapelière. Histoire du mouvement ouvrier dans la chapellerie, Paris, Domat-Montchrestien, 1941, 447 p., p. 52.

${ }^{64}$ Ortiz (Vincent), Un chapeau de paille de Septfonds..., ouv. cité, pp. 24-28. 
Pour le feutre de poils, spécialité toulousaine et aixoise, les étapes successives sont très différentes ${ }^{65}$. La phase initiale joue un grand rôle, les peaux doivent être préparées et classées, puis elles subissent le secrétage, à base de nitrate de mercure, qui confère des propriétés feutrantes aux poils. Ces derniers sont ensuite coupés avant d'être foulés. Le foulage se subdivise lui-même en arçonnage, semoussage, travail de la foule et dressage pour aboutir à une ébauche de forme. Le ponçage, la teinture et l'appropriage - destiné à affermir et rendre étanche le chapeau - parachèvent le tout. Pour les feutres de laine, apprêteurs, fouleurs et approprieurs constituent également des métiers spécifiques au milieu du XIX ${ }^{\mathrm{e}}$ siècle à Espéraza $^{66}$. À l'intérieur de ces spécialités, des spécificités se développent, à l'image de la seule préparation des peaux qui requiert les interventions successives des planeurs, des arracheuses, des repasseuses et des coupeuses ${ }^{67}$. L'utilisation de quatre types de poils différents vient complexifier cette division des tâches. En effet, les poils de castor et de loutre restent réservés aux chapeaux de luxe extra-fins, ceux de lièvre occupent une place médiane, mais les chapeliers du Sud travaillent surtout le poil de lapin destiné à la confection des chapeaux courants dont la demande explose. Le caractère très agricole des matières premières, paille et poils de lapin, explique en partie la "ruralisation » de l'activité chapelière $^{68}$, mais l'efficacité et la souplesse de la main-d'œuvre paysanne y contribuent également.

En effet, les gains de productivité obtenus lors de la division du travail se doublent de salaires bas et payés aux pièces à des travailleurs qui conçoivent ces revenus comme un supplément bienvenu. Pendant l'essentiel du XIX $\mathrm{X}^{\mathrm{e}}$ siècle, les ouvriers chapeliers d'Espéraza, Nay, ou Septfonds, conservent des liens privilégiés avec la terre. À Espéraza, parmi les 213 ouvriers chapeliers recensés sous le Second Empire, 102 possèdent une cote cadastrale et parmi ceux-ci 65 disposent de moins d'un hectare ${ }^{69}$. Ces micro-propriétaires doivent exercer une activité complémentaire pour rester sur leur terre et éventuellement reconstituer un patrimoine foncier viable. Mais ces chiffres ne prennent pas en compte ceux qui besognent à domicile occasionnellement et qui préfèrent se déclarer «agriculteurs ». À Caussade, les observateurs de la Belle Époque évoquent couramment la présence de plus de 1000 chapeliers dans le bourg, alors que le recensement de 1901 n'en dénombre que $200^{70}$. Cette sous-estimation criante confirme la discrétion d'une industrie en grande partie composée de « mains invisibles ».

\footnotetext{
${ }^{65}$ Passama (Julie), La chapellerie toulousaine ..., ouv. cité, pp. 13-29.

${ }^{66}$ Jullian (Antoine), Itinéraires et changements sociaux dans la chapellerie espérazanaise..., ouv. cité, p. 45.

${ }^{67}$ Passama (Julie), La chapellerie toulousaine ..., ouv. cité, p. 16.

${ }^{68}$ Cette importance des ressources locales se retrouve dans les 48 petites chapelleries du Var, dont celle de Vidauban qui emploie 34 ouvriers. Ces dernières utilisent essentiellement la laine d'agneau, le poil de lapin et celui de veau au milieu du $\mathrm{XIX}^{\mathrm{e}}$ siècle, d'après Noyon, Statistique du département du Var, Draguignan, Imprimerie H. Bernard, 1848 (?), 646 p., p. 636. La plupart de ces établissements remontent au début du XIX ${ }^{\mathrm{e}}$ siècle comme l'indique la sous-série $16 \mathrm{M}$ des archives départementales du Var.

${ }^{69}$ Martin (Danielle), Naissance et développement du mouvement ouvrier dans l'Aude. Les ouvriers chapeliers d'Espéraza vers le milieu du XIX $X^{e}$ siècle, mémoire de maîtrise, université de Toulouse 2-Le Mirail, 1973, 112 p., pp. 49-51.

${ }^{70}$ Ortiz (Vincent), Un chapeau de paille de Septfonds..., ouv. cité, pp. 53-54.
} 
La persistance du travail à façon rend également caducs les salaires quotidiens établis pour la statistique nationale. À Caussade, la situation industrielle de 1896 précise que «le plus grand nombre » des ouvrières est rétribué aux pièces ${ }^{71}$. Ces travailleuses étant pour l'essentiel des jeunes filles de moins de 40 ans, leur rétribution devient extrêmement faible selon les canons de l'époque qui la fixe généralement à moins de la moitié de celle des hommes adultes. Enfin, le donneur d'ordres ne peut que se féliciter d'une production à domicile auto-contrôlée, car la réputation de «bonne tresseuse » ou de « bonne couseuse » de toute une famille est en jeu. Ultime avantage, ce système ne nécessite ni contremaîtres, ni vastes bâtiments ; à Septfonds, seul l'établissement de Fortuné Cantecor peut passer pour une usine. Malgré cela, les nombreux petits industriels ruraux n'hésitent pas à introduire des machines adaptées à certaines étapes du processus de production, sans se lancer dans des investissements démesurés.

\section{Une mécanisation légère et faiblement motorisée}

La machine à coudre à pédale symbolise cette évolution douce, installée dans de petits ateliers, ou à domicile, elle permet de coudre quarante à cinquante chapeaux de paille par jour au lieu d'une dizaine à la main ${ }^{72}$. Les premières apparaissent dans les années 1860 . La mécanique du Français Legat, introduite à Septfonds dès 1875, se montre « supérieure à toutes les machines anglaises et américaines car il est impossible de faire une différence entre la couture de cette machine et celle faite à la main ${ }^{73}$. Un chapeau ordinaire prenant quinze mètres de tresse, est confectionné sans interruption par celle-ci en dix minutes ${ }^{74}$. Les chapeliers du village de Septfonds acquièrent cet engin révolutionnaire avant les Toulousains $^{75}$. En 1902, les ateliers de Septfonds et Caussade abritent 2000 machines à coudre de types variés ${ }^{76}$.

La diffusion de la dresseuse mécanique de Mathias et Legat, mise au point en 1867, est encore plus rapide. Elle apparaît dès 1868 à Septfonds, puis dans les années 1880 à Toulouse $^{77}$. Cette machine se compose d'un moule sur lequel l'ébauche de chapeau est emboîtée. Dans un second temps, un couvre-moule doté d'une membrane en caoutchouc déformable vient épouser la forme du moule inférieur en comprimant le chapeau grâce à de l'eau chaude sous pression circulant derrière la membrane. Un seul ouvrier avec cette dresseuse mécanique peut effectuer le travail de quarante personnes en dressant 400 chapeaux par jour ${ }^{78}$. Cette machine peut recevoir une très grande variété de moules grâce à la souplesse du caoutchouc. Mais elle se révèle chère à l'achat et les petits chapeliers du Sud lui préfèrent des presses à pédale ou hydrauliques dans lesquelles les ébauches sont mises en forme entre deux pièces métalliques chauffées à la vapeur, au gaz ou à la gazoline ${ }^{79}$. En 1902, Septfonds

\footnotetext{
${ }^{71}$ Archives départementales du Tarn-et-Garonne, 6M 899 et $10 \mathrm{M} 1410$.

${ }^{72}$ Gebelin (Jacques), Une grande industrie du Bas-Quercy..., ouv. cité, p. 8.

${ }^{73}$ Vial (Jean), La coutume chapelière. Histoire du mouvement ouvrier dans la chapellerie, Paris, F. Loviton, 1941, 451 p., p. 104.

${ }^{74}$ Passama (Julie), La chapellerie toulousaine ..., ouv. cité, p. 100.

${ }^{75}$ Ibidem

${ }^{76}$ Doumerc, Industrie, commerce ..., ouv. cité, p. 19.

${ }^{77}$ Gebelin (Jacques), Une grande industrie du Bas-Quercy..., ouv. cité, p. 8

${ }^{78}$ Vial (Jean), La coutume chapelière ..., ouv. cité, p. 107.

${ }^{79}$ Doumerc, Industrie, commerce ..., ouv. cité, p. 19
} 
et Caussade disposent ainsi de deux cents presses très diverses ${ }^{80}$. Dès les années 1880 , le Septfontois Fortuné Cantecor achète une machine à vapeur et fait fonctionner son usine par un système de haute et basse pression exceptionnel en France, puis il adopte les premières presses à gaz ${ }^{81}$. Enfin, dans les années 1860 , avec seulement quelques années de retard sur les Américains, deux chapeliers en feutre toulousains s'équipent de "bâtisseuses ${ }^{82}$. Ce sont des ventilateurs enfermés dans des tambours et destinés à coller le poil de lapin sur la forme ${ }^{83}$. L'une de ces bâtisseuses a été conçue par un industriel-mécanicien local, Edmond de Planet. Elle peut faire le travail de sept à huit ouvriers.

Toutefois, l'utilisation des machines demeure modeste jusqu'en 1890, leur vulgarisation s'effectuant seulement à partir de cette date. L'arrivée de l'électricité facilite ce processus en permettant de conserver la dynamique du travail à domicile, ou en petits ateliers, avec des mécaniques simples. L'interdiction du travail usinier de nuit pour les femmes, par la loi de 1892, stimule également la mécanisation dans les usines tout en maintenant l'intérêt d'un système de production dispersé intégrant l'activité domestique féminine. En effet, beaucoup de passes délicates et décisives comme le garnissage, ne sont pas mécanisables. La haute vallée de l'Aude connaît la même chronologie et un dualisme des structures de production identique, " la ponceuse, en particulier, permet de préparer des chapeaux en quelques minutes, alors qu'il fallait autrefois près d'une heure ${ }^{84}$. Dans l'industrie béarnaise du béret, il faut attendre les années 1920 pour que les machines manuelles soient remplacées progressivement par de véritables tricoteuses à diminutions automatiques capables de réaliser et d'assembler les vingt-quatre sections triangulaires du béret et son ourlet ${ }^{85}$. Les étapes suivantes du foulonnage, du feutrage, de l'enformage, du séchage, du grattage, du rasage et du garnissage deviennent également de moins en moins manuelles ${ }^{86}$.

Au total, initiée par des dynamiques commerciales, la chapellerie méridionale a su s'adapter aux exigences quantitatives et qualitatives du marché mondial de la mode né pendant le $\mathrm{XIX}^{\mathrm{e}}$ siècle. Profondément implantées dans les sociétés locales, en particulier rurales, les structures dispersées de production font preuve d'une exceptionnelle réactivité qui les conduisent au triomphe. Le village de Septfonds devient le grand centre français de la chapellerie de paille avec plus de dix millions d'unités produites annuellement à la veille de la Première Guerre mondiale ${ }^{87}$ et un chiffre d'affaires qui dépasse les six millions de francs ${ }^{88}$. Espéraza atteint son apogée en 1929 avec seize usines fabricant vingt-quatre millions de

\footnotetext{
${ }^{80}$ Ibidem.

${ }^{81}$ Ibidem.

${ }^{82}$ Archives municipales de Toulouse, Exposition des produits des Beaux Arts et de l'Industrie à Toulouse en 1865, Toulouse, Viguier, 1866, 855 p., p. 93

${ }^{83}$ Archives départementales de la Haute-Garonne, 5 M 12, liasse A.

${ }^{84}$ Gayda (Raymond), L'industrie chapelière dans la haute vallée de l'Aude, thèse de droit, université de Toulouse 1, 1947, 161 p., pp. 37-38.

${ }^{85}$ Jouvion (Philippe), Le béret, Rodez, Éditions du Rouergue, 1998, 136 p., pp. 52-53.

${ }^{86}$ Ibidem, pp. 54-55.

${ }^{87}$ Cambon (Claudine), La chapellerie en Tarn-et-Garonne, mémoire de maîtrise, université de Toulouse 2-Le Mirail, 1979, 75 p., p. 16. Ce chiffre correspond à une production de sept millions de chapeaux à Septfonds et plus de trois millions à Caussade, c'est-à-dire plus de la moitié de la production française de chapeaux de paille

${ }^{88}$ Doumerc, Industrie, commerce ..., ouv. cité, p. 18.
} 
chapeaux et de cloches, dont neuf millions sont exportés ${ }^{89}$. Ce bourg d'un peu plus de deux mille habitants, associé aux localités voisines, constitue alors le deuxième pôle mondial de la chapellerie de feutre après Monza en Italie ${ }^{90}$. Enfin, entre 1920 et 1959, Oloron-Sainte-Marie s'affirme comme la capitale mondiale du béret avec huit usines sur les vingt-et-une que compte la France à l'aube des années soixante ${ }^{91}$. Cinq autres fabriques se trouvent dans la petite ville voisine de Nay, confirmant ainsi la prédominance du pôle béarnais.

${ }^{89}$ Jullian (Antoine), Itinéraires et changements sociaux dans la chapellerie espérazanaise..., ouv. cité, p. 7 .

${ }^{90}$ Ibidem.

${ }^{91}$ Jouvion (Philippe), Le béret..., ouv. cité, p. 52. 